\title{
Effect of sildenafil citrate on women affected by sexual dysfunction referred to health clinics
}

\author{
SANAZ ZEINALZADEH ${ }^{1, B, D, F}$, MARZIEH AKBARZADEH ${ }^{2, A, D-G}$, POUYA FARIDI ${ }^{3, D, G}$, \\ ABDOL ALI MOHAGHEGHZADEH ${ }^{3, A, G}$, MEHRAB SAYADI ${ }^{4, C, D}$
}

\author{
${ }^{1}$ Department of Midwifery, School of Nursing and Midwifery, Shiraz University of Medical Sciences, Shiraz, Iran \\ ${ }^{2}$ Maternal - Fetal Medicine Research Center, Department of Midwifery, School of Nursing and Midwifery, Shiraz \\ University of Medical Sciences, Shiraz, Iran \\ ${ }^{3}$ Department of Traditional Pharmacy, School of Pharmacy, Shiraz University of Medical Sciences, Shiraz, Iran \\ ${ }^{4}$ Student Research Committee, School of Medicine, Department of Biostatistics, Shiraz University of Medical \\ Sciences, Shiraz, Iran
}

A - Study Design, B - Data Collection, C - Statistical Analysis, D - Data Interpretation, E - Manuscript Preparation, F - Literature Search, G - Funds Collection

Summary Background. Female sexual dysfunction is a serious, multifactorial problem which affects women's quality of life. Objectives. This study aimed at assessing the effect of sildenafil citrate on sexual dysfunction among women of reproductive age. Materials and methods. The present randomized clinical trial was conducted on 84 married women between 18 and 40 years old who suffered from sexual dysfunction. The inclusion criteria of the study were being married and between 18-40 years old, obtaining scores $\geq 22$ in FSFI, not having a history of hypothyroidism or hyperprolactinemia, and not suffering from dyspareunia or vaginismus. The intervention group was required to consume $50 \mathrm{mg}$ sildenafil citrate 1 hour before sexual intercourse for 5 weeks, and the control group received placebo. FSFI was completed by both study groups before and 5 weeks after the treatment. The data were analyzed through SPSS (v. 18) and paired $t$-test and independent $t$-test.

Results. Most of the women (67.2\%) were between 30 and 40 years old. The study results revealed a significant difference between the two groups regarding the mean scores of sexual desire $(p=0.011)$, sexual arousal $(p=0.001)$, lubrication $(p=0.026)$, orgasm and sexual satisfaction $(p<0.001)$, and the whole dimension $(p<0.001)$. Besides, total mean sexual function before and after for the intervention and control groups was $21.1 \pm 3.28$ vs. $17.22 \pm 5.9(p<0.001)$.

Conclusion. In this study, sildenafil citrate was effective in enhancing sexual desire, arousal, orgasm, and sexual satisfaction. Of course, more studies need to be conducted on this issue.

Key words: female, sexual, dysfunction, sildenafil citrate.

Zeinalzadeh S, Akbarzadeh M, Faridi P, Mohagheghzadeh AA, Sayadi M. Effect of sildenafil citrate on women affected by sexual dysfunction referred to health clinics. Fam Med Prim Care Rev 2017; 19(2): 167-172, doi: https://doi.org/10.5114/fmpcr.2017.67873.

\section{Background}

Female sexual dysfunction is a common multifactorial health problem which is mostly neglected in the general population while affecting women's quality of life [1]. In the study conducted by Zhang conducted in Hong Kong in 2007, 1510 Chinese women between 19 and 49 years old were interviewed; $37.9 \%$ of them showed at least one type of sexual dysfunction [2]. Moreover, Hassanin et al. performed a study in Egypt and reported the prevalence of female sexual dysfunction to be 76.9\%. Besides, each woman had at least one sexual disorder, with low sexual desire being the most prevalent one (66.4\%). Also, as the women's age increased, the prevalence of sexual problems increased as well [3]. In Iran also, several research studies have been conducted on women's sexual function. However, most of the studies have focused on the prevalence and types of sexual disorders, and less attention has been paid to treatment dimensions, including education, consultation, and pharmacotherapy. For instance, Safarinejad carried out research on 20-60-year-old women in different provinces of the country, and reported the prevalence of low sexual desire, lack of sexual arousal, lack of orgasm, and pain during intercourse as $35 \%, 30 \%, 37 \%$, and $26 \%$, respectively [4]. Furthermore, Ghanbarzadeh et al. conducted a study on the prevalence of sexual dysfunction among 15-72-year-old women (mean age: $31.5 \pm$ 9.1 years) in Birjand. The study results showed that out of the 821 subjects under study, 694 (84.6\%) had active sexual intercourse with their husbands, but $239(29.1 \%)$ had less than one intercourse per week. In addition, 320 participants (39\%) were not satisfied with their sexual activity, and $86(10.5 \%)$ had never experienced orgasm [5].

In general, women's sexual function is affected by a large number of biological, psychological, and socio-cultural factors. Nevertheless, only $20 \%$ of women and $10-30 \%$ of men suffering from sexual dysfunction refer to physicians for treatment [6]. In spite of the importance of sexual dysfunction in females, limited treatment methods, such as psychotherapy (marital consultation and training), pharmacotherapy (hormone therapy and PDE5 inhibitors), and complementary medicine (herbal medicine) are available.

Sildenafil citrate is a selective inhibitor of cGMP-specific PDE5, and is effective in the treatment of erectile dysfunction in males [7]. In addition to playing physiological roles in males' sexual responses, this medication can also be effective in females' sexual arousal [8]. Sildenafil citrate is also available under the commercial names Viagra and Revatio.

Sexual dysfunction leads to couples' psychological vulnerability and disturbs their quality of life. Midwives, being among 
the most important members of the health and treatment team, can assist women regarding their concerns about sexual issues at various stages of life. Therefore, considering the community-based responsibility of midwives, major steps can be taken towards improving sexual relationships, empowering the psychological dimensions, and strengthening the family base.

\section{Objectives}

The present study aims to investigate the effect of sildenafil citrate on women's sexual dysfunction.

\section{Material and methods}

The research was approved by the Ethics Committee of Shiraz University of Medical Sciences, and was registered in the Iranian registry of clinical trials. The present randomized clinical trial was conducted on 84 women between 18 and 40 years old who suffered from sexual dysfunction and had referred to the clinics affiliated to the Enghelab health center, Shiraz, Iran.

According to the type and objectives of the study, and considering type I error $(\alpha)$ of $5 \%$, power of $80 \%$, minimum mean difference of 0.6 , variance of 0.92 , and the following formula, 36 subjects agreed to participate in each study group,

$$
n=2\left(z_{1-\alpha / 2}+z_{1-\beta}\right) \delta / \mathrm{d}^{2} .
$$

Nonetheless, since the present study was a longitudinal one, considering the sample drop-out rate of $20 \%$ and using the following formula, an 84 -subject sample size (42 subjects in each group) was selected for the study (Figure 1), $n^{\prime}=n \times 1(1-p)$.
The inclusion criteria of the study were being willing to take part in the study, being married, being between 18 and 40 years old, obtaining scores $\geq 22$ in the Female Sexual Function Index (FSFI), not being pregnant, not having a history of myocardial infarction or cardiovascular disease, not having a history of uncontrolled hypertension or hypotension, not using drugs affecting sexual function, such as common antidepressants, not using hormone drugs, particularly oral contraceptive pills, not using alcohol or drugs, not having a history of active peptic ulcer, not having a history of headaches such as migraine, and not suffering from dyspareunia or vaginismus.

The study data were gathered using the FSFI questionnaire and a demographic information form.

In cases where the samples obtained scores $\geq 22$, TSH and prolactin levels were tested in order to reject thyroid and prolactin disorders, which are among the secondary causes of sexual dysfunction.

In this study the samples were selected through convenience sampling and randomly divided into an intervention and a control group. The subjects were required to consume the medications one hour before sexual intercourse.

For the interventional group sildenafil citrate tablets $50 \mathrm{mg}$ were used for 4 weeks, one hour before intercourse. For the control group, if the subject wished to participate, had completed the consent form, and had the criteria for inclusion, they were enrolled in the study. The control group received placebo for 35 days, two tablets every 12 hours. All the samples were contacted weekly by Short Messages (SMS) and phone calls to encourage them to consume the drugs.

The FSFI questionnaire, with 19 items in the form of self-report in six independent areas, measures female sexual function, including sexual desire, mental stimulation, humidity, orgasm,

\section{Enrollment}

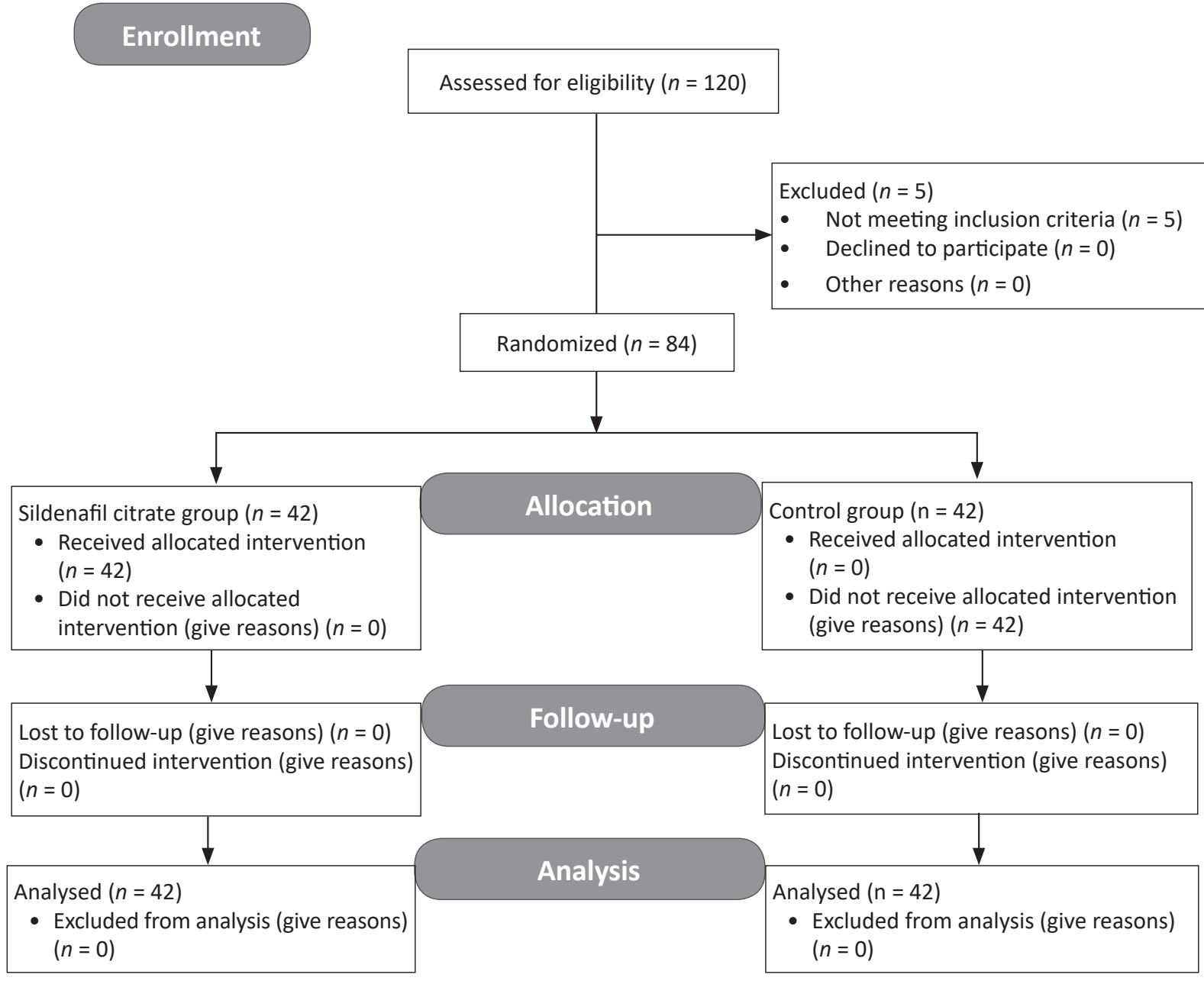

Figure 1. Flow diagram of sampling 


\begin{tabular}{|c|c|c|c|c|c|}
\hline \multicolumn{2}{|c|}{ Areas of sexual function } & Sildenafil Citrate group $(n=41)$ & Control group $n=42$ ) & $T$-value & $p$ \\
\hline \multirow[t]{3}{*}{ Sexual desire } & Before intervention & $3.15 \pm 0.94$ & $2.78 \pm 0.88$ & 1.85 & 0.067 \\
\hline & After intervention & $3.51 \pm 0.88$ & $2.97 \pm 1.01$ & 2.60 & 0.011 \\
\hline & $p$-value & 0.001 & 0.208 & & \\
\hline \multirow[t]{3}{*}{ Sexual arousal } & Before intervention & $3.39 \pm 0.82$ & $3.11 \pm 1.17$ & 1.25 & 0.214 \\
\hline & After intervention & $4.05 \pm 0.82$ & $3.22 \pm 1.24$ & 3.56 & 0.001 \\
\hline & $p$-value & $<0.001$ & 0.377 & & \\
\hline \multirow[t]{3}{*}{ Lubrication } & Before intervention & $4.18 \pm 0.89$ & $3.75 \pm 1.45$ & 1.65 & 0.103 \\
\hline & After intervention & $4.65 \pm 0.82$ & $4.04 \pm 1.54$ & 2.27 & 0.026 \\
\hline & $p$-value & 0.501 & 0.177 & & \\
\hline \multirow[t]{3}{*}{ Orgasm } & Before intervention & $3.48 \pm 0.94$ & $3.18 \pm 1.19$ & 1.29 & 0.199 \\
\hline & After intervention & $4.15 \pm 0.89$ & $3.23 \pm 1.36$ & 3.63 & $<0.001$ \\
\hline & $p$-value & $<0.001$ & 0.715 & & \\
\hline \multirow[t]{3}{*}{ Sexual satisfaction } & Before intervention & $4.25 \pm 0.8$ & $3.62 \pm 1.37$ & 2.56 & 0.012 \\
\hline & After intervention & $4.73 \pm 0.78$ & $3.74 \pm 1.65$ & 3.50 & $<0.001$ \\
\hline & $p$-value & $<0.001$ & 0.515 & & \\
\hline
\end{tabular}

\begin{tabular}{|c|c|c|c|c|}
\hline \multicolumn{2}{|l|}{ Areas of sexual function } & Sildenafil Citrate group & Control group & $p$ \\
\hline \multirow[t]{3}{*}{ Sexual function (total) } & Before intervention & $18.47 \pm 3.22$ & $16.45 \pm 4.9$ & 0.028 \\
\hline & After intervention & $21.1 \pm 3.28$ & $17.22 \pm 5.9$ & $<0.001$ \\
\hline & $p$-value & $<0.001$ & 0.229 & \\
\hline
\end{tabular}

\begin{tabular}{|c|c|c|c|c|}
\hline \multicolumn{2}{|l|}{ Areas of sexual function } & \multirow{2}{*}{\begin{tabular}{|l} 
Sildenafil Citrate group \\
$n(\%)$ \\
\end{tabular}} & \multirow{2}{*}{$\begin{array}{l}\text { Control group } \\
n(\%)\end{array}$} & \multirow{3}{*}{$\begin{array}{l}p \\
0.028\end{array}$} \\
\hline \multirow{4}{*}{ Sexual function (total) } & & & & \\
\hline & Before intervention & $41(97.6)$ & $42(100)$ & \\
\hline & After intervention & $23(54.8)$ & $34(81)$ & $<0.001$ \\
\hline & $p$-value & $<0.001$ & 0.229 & \\
\hline
\end{tabular}

satisfaction, and sexual pain measures. Based on their sexual performance and feeling, the women responded to the questions during the last 4 weeks. FSFI has been used in research as a valid instrument to assess sexual function [9-11]. The reliability of this scale was obtained as 0.70 . The reliability and validity indexes reported by Mohammadi et al. (2008) are the basis of the present study [12].

The FSFI questionnaire was completed by the study participants before and 5 weeks after the consumption of the medication. Finally, the data were entered into the SPSS statistical software (v. 18), and for analysis between groups we used the independent $t$-test, and for internal analysis in each group before and after the intervention we used the paired $t$-test. $P<0.05$ was considered as statistically significant.

\section{Results}

In this study, most of the subjects (67.2\%) were between 30 and 40 years old, and $32.8 \%$ were within the $20-30$ year-old-age range. Before the intervention, the mean of TSH $(2.6 \pm 1.07)$ and prolactin levels $(10.05 \pm 5.27)$ were normal in both the intervention and control groups. The study results revealed a significant difference between the two groups regarding the mean scores of sexual desire $(p=0.011)$, sexual arousal $(p=0.001)$, lubrication ( $p=0.026)$, orgasm and sexual satisfaction $(p<0.001)$, and the whole dimension $(p<0.001)$. The percentage of changes was $0.35 \pm 0.65$ vs. $0.18 \pm 0.94$ in sexual desire, $0.65 \pm 0.71$ vs. $0.11 \pm 0.83$ in sexual arousal, $0.47 \pm 0.81$ vs. $0.29 \pm 1.38$ in lubrication, $0.66 \pm 0.89$ vs. $0.05 \pm 1$ in orgasm, $0.47 \pm 0.63$ vs. $0.11 \pm$
1.1 in sexual satisfaction, and $2.62 \pm 2.72$ vs. $0.76 \pm 4.05$ in the whole dimension (Table 2 and 3 ).

According to the study results, no significant difference was found between the two groups regarding the mean score of the state of anxiety before the intervention $(p=0.67)$; however, the two groups were revealed to be significantly different in this regard after the intervention $(p=0.026)$. On the other hand, no significant difference was observed between the two groups regarding their mean scores of anxiety trait before $(p=0.908)$ and after the intervention $(p=0.088)$. Therefore, the frequency of sexual dysfunction decreased to $54.8 \%$ from $97.6 \%$ after the intervention (Table 3).

\section{Discussion}

The cycle of females' sexual responses includes five phases of arousal, stability, interest, orgasm, and resolution. These phases are influenced by various psychological, environmental, and physiological (hormonal, vascular, muscular, and nervous) factors. Also, each of these stages can be affected by age, disease, using medications, alcohol abuse, drug abuse, and cultural status of the society, eventually leading to change in the quality of marital relationships. Moreover, disorders in any of the above-mentioned phases can result in an increase in the incidence of sexual dysfunction [13].

In this study, the mean levels of TSH and prolactin were normal in both study groups before the intervention. Kadioglu et al. (2005) investigated sexual dysfunction in patients suffering from hyperprolactinemia, and the results revealed sexual dysfunction in 22 out of the 25 patients $(88 \%)$. On the other hand, only 4 
out of the 16 control subjects (25\%) had sexual dysfunction [14]. Similarly, Atis et al. reported the prevalence of sexual dysfunction to be higher among women with hypothyroidism and subclinical hypothyroidism compared to the control group [15].

The findings of the current study revealed a significant difference between the two groups regarding their sexual desire mean scores ( $3.51 \pm 0.88$ vs. $2.97 \pm 1.01)$. In a placebo-controlled study in the U. S., 202 menopausal women with Female Sexual Arousal Dysfunction (FSAD) were divided into two groups, receiving either sildenafil citrate $(50 \mathrm{mg})$ or a placebo [16]. The study results showed that sildenafil citrate was more effective than the placebo in the women with FSAD but not suffering from Hypoactive Sexual Desire Disorder (HSDD) $(p<0.02)$. On the other hand, no significant change was observed in the sexual function of the women who suffered from both HSDD and FSAD. Berman et al. also mentioned sildenafil citrate as an appropriate treatment for women suffering from FSAD but not HSDD. In that study the potential causes of FSAD were not comprehensively investigated. The existence of psychological or nervous disorders which might have affected the women's sexual function was not determined, either. Overall, the findings of the study by Berman et al. might not be reliable due to the fact that the women suffering from nervous disorders might have had sexual desire but did not experience sexual arousal. Alexander et al. reported that these women could be treated with sildenafil citrate [17].

Furthermore, in a double-blind, placebo-controlled study which was conducted on the sexual function of 19 women with Spinal Cord Injury ( $\mathrm{SCl}$ ), the participants were randomly divided into sildenafil and placebo groups. Then, vaginal hyperemia was assessed using plethysmography. The findings of that study showed that the scores of mental arousal significantly increased in the sildenafil group $(p<0.01)$. Yet, a more significant improvement was observed in the subjects' sexual function when the administration of sildenafil citrate was accompanied by visual stimulation and a guide booklet. Although a greater increase in vaginal hyperemia was detected in the sildenafil group, no significant difference was observed between the two groups in this regard ( $p$ $=0.07)$. Moreover, Sipski et al. showed that sildenafil could relatively improve the sexual function in women suffering from $\mathrm{SCl}$ [18], which is consistent with the findings of the present study.

According to the results of the current study, the mean score of sexual stimulation increased from $3.39 \pm 0.82$ to $4.05 \pm 0.82$ in the intervention group $(p<0.001)$. Salerian et al. [19]. conducted a study on 61 males and 31 females who had referred to psychiatry outpatient clinics, and used sildenafil (12.5-100 $\mathrm{mg}$ ) for treating sexual disorders resulting from psychological disease. The results of the study showed a significant increase in both males' and females' scores of arousal, sexual desire, orgasm, and sexual satisfaction. The increase in the females' sexual desire in that study seems to be a psychological reaction to receiving sildenafil. Previous studies had also shown that response to placebo could occur due to the individuals' psychological reaction. Therefore, improvement of the study subjects' sexual desire could have occurred due to the fact that they expected a positive change after using sildenafil. Another study also showed that, in comparison to the placebo, sildenafil was more effective in increasing clitoral blood flow [20].

In the present study, a significant difference was found between the two groups regarding lubrication before $(p=0.016)$ and after the intervention $(p=0.001)$. Ferrara and Zaslau (2007) used sildenafil citrate in order to treat sexual dysfunction in women suffering from neurogenic injury (L5-S1 disk herniation). The data were gathered using the FSFI questionnaire, and showed that the females' sexual function score increased from 48 to 57 . Thus, the findings of that study showed that sildenafil citrate increased the subjects' sexual stimulation and lubrication $(p<0.001)[21]$

Similarly, Caruso showed that sildenafil could improve arousal, lubrication, orgasm, sexual satisfaction, and painful in- tercourse in women suffering from type I diabetes [22]. In general, desirable sexual response in females is accompanied by increase in vaginal blood flow. Ferrara, Zaslau, and Caruso also revealed the positive effect of sildenafil citrate through inhibition of PDE5 and increase of nitric oxide production, eventually increasing vaginal blood flow and lubrication.

The effect of using sildenafil citrate on improving women's quality of life might be based on the assumption that males and females have similar sexual response cycles. Therefore, using sildenafil increases vaginal vasocongestion and affects females' mental sexual arousal [23].

In the current study, the intervention group's orgasm mean score increased from $3.48 \pm 0.94$ before the intervention to 4.15 \pm 0.89 after the intervention, and was significant in comparison to the control group. Caruso investigated the effect of sildenafil citrate in 68 healthy women between 19 and 38 years old who had no signs of sexual disorders. The subjects were required to consume either sildenafil or the placebo for 4 weeks, and to complete the Personal Experience Questionnaire (PEQ). In comparison to the placebo, sildenafil was shown to be more effective in improving arousal $(p<0.01)$, orgasm $(p<0.001)$, and sexual satisfaction $(p<0.001)$ which is in agreement with the findings of the present study [24].

Furthermore, Dasgupta et al. assessed the effect of sildenafil citrate on the treatment of secondary sexual dysfunction in women with multiple sclerosis. However, sildenafil showed no effects, which is in contrast to the present study results [25].

Sildenafil's main mechanism of action is increasing the effect of nitric oxide by the inhibition of cGMP enzymatic destruction [26]. The specific-PDE5 inhibitor leads to the deactivation of cGMP in both the clitoris and the penis. In addition to the vagina, PDE5 is also present in the clitoris and the smooth muscles of the labia. Consumption of sildenafil results in hyperpolarization and relaxation of the tissue through the above-mentioned mechanism, and eventually leads to increase in blood flow, hyperemia, and inflation $[27,28]$. Thus, it is highly effective in females' sexual function, particularly sexual arousal [29]. Its effects on increasing sexual arousal and lubrication [30], and loosening of the smooth muscles, particularly in arteries, veins, and arterioles of the vagina and the clitoris have also been reported in other studies [31].

In this study, the sexual satisfaction mean score in the intervention group increased from $4.25 \pm 0.8$ before the intervention to $4.73 \pm 0.78$ after the intervention, which was significantly different from the control group $(p<0.001)$. Ichikawa et al. evaluated the sexual satisfaction of women whose husbands had received sildenafil citrate due to erectile dysfunction. The results showed the effectiveness of the treatment in $90 \%$ of the sexual partners. Also, the partners' life quality was reported to have improved in $60 \%$ of the women under study. In that study, most of the subjects $(66.7 \%)$ were satisfied, and $20 \%$ were not satisfied with the treatment with sildenafil. Besides, only $20 \%$ of the females' partners were worried about the side effects [32]. These findings were in line with those of the present study.

However, our study results are in contrast to those of a study by Basson et al., in which they randomly used 10-100 mg sildenafil for 577 women with natural estrogen and 204 with estrogen deficiency who complained of FSAD. The initial presentation of the treatment was detected in $46 \%$ and $50 \%$ of the women with natural and deficient estrogen, respectively. The study results revealed no significant difference between sildenafil citrate and the placebo. Thus, further studies are recommended to investigate the physiological effects of sildenafil citrate on the genital system in order to improve the sexual response in women with various types of sexual dysfunction [33].

It should be noted that sildenafil citrate has been used for a long time in the treatment of male sexual dysfunction, and the application of sildenafil in the treatment of arousal disorder in women is still controversial. Lo Monte et al. recommended that 
sildenafil citrate should be used as the last treatment option, and say that proper diagnosis and treatment of female sexual disorders requires a thorough history of sexual, psychological, and medical consultation with a specialist [34].

Besides, the effectiveness of sildenafil citrate in in vitro studies on smooth-muscle relaxation in clitoral tissue leads the authors to predict that it may be effective in sexual arousal disorder, multiple sclerosis, diabetes or antidepressant [35].

In another study, the effect of sildenafil citrate on genital engorgement disorder in 90 premenopausal women with female sexual arousal was evaluated. The intervention group received 100 $\mathrm{mg}$ drug daily and were compared with the placebo group. The effectiveness was measured by MRI. The results showed that in the case group's clitorises engorgement did not happen, so sildenafil sexual response in women with FSAD was not strengthened [36].

\section{Conclusions}

The findings of the present study showed that sildenafil citrate was effective in enhancing sexual desire, arousal, orgasm, and sexual satisfaction. However, considering the study sample size, more studies need to be conducted on the issue.

Acknowledgements. This article is a part of a thesis by Sanaz Zeinalzadeh, (thesis number: 91-6o16, IRCT: 201205219818N1). The researchers would like to thank the Research and Technology Department of Shiraz University of Medical Sciences. The authors would like to thank Shiraz University of Medical Sciences, Shiraz, Iran, and also the Center for the Development of Clinical Research of Nemazee Hospital, and Dr. Nasrin Shokrpour for editorial assistance.

Source of funding: This article is a part of a thesis by Sanaz Zeinalzadeh (thesis number: 91-6016, IRCT: 201205219818N1). Conflicts of interest: The authors declare no conflicts of interest.

\section{References}

1. Basson R. Women's sexual dysfunction: revised and expanded definitions. CMAJ 2005; 172(10): 1327-1333. Review.

2. Zhang H, Yip PS. Female sexual dysfunction among young and middle-aged women in Hong Kong: prevalence and risk factors. $J$ Sex Med 2012; 9(11): 2911-2918.

3. Hassanin IM, Helmy YA, Fathalla MM, et al. Prevalence and characteristics of female sexual dysfunction in a sample of women from Upper Egypt. Int J Gynaecol Obstet 2010; 108(3): 219-223.

4. Safarinejad M. Female sexual dysfunction in a population-based study in Iran: prevalence and associated risk factors. Int J Impot Res 2006; 18(4): 382-395.

5. Ghanbarzadeh N, Nadjafi-Semnani M, Ghanbarzadeh MR, et al. Female sexual dysfunction in Iran: study of prevalence and risk factors. Arch Gynecol Obstet 2013; 287(3): 533-539.

6. Aslan E, Beji NK, Gungor I, et al. Re: Prevalence and risk factors for low sexual function in women: a study of 1,009 women in an outpatient clinic of a university hospital in Istanbul-a response. J Sex Med 2009; 6(5): 1491-1492.

7. Khan MA, Mikhailidis DP, Morgan RJ. Sildenafil: urologist's view. Curr Med Res Opin 2000; 16(Suppl. 1): S48-S58.

8. D’Amati G, di Gioia CR, Bologna M, et al. Type 5 phosphodiesterase expression in the human vagina. Urology 2002; 60(1): 191-195.

9. Rosen RC. Assessment of female sexual dysfunction. Review of validated methods. Fertil Steril 2002; 77(Suppl. 4): S89-S93. Review.

10. Nowosielski K, Wróbel B, Sioma-Markowska U, et al. Development and validation of the Polish Version of the Female Sexual Function Index in the Polish population of females. J Sex Med 2013; 10(2): 386-395.

11. Isidori AM, Pozza C, Esposito K, et al. Development and Validation of a 6-Item Version of the Female Sexual Function Index (FSFI) as a diagnostic tool for female sexual dysfunction. J Sex Med 2009; 7(3): 1139-1146.

12. Mohammadi H, Heidari M, Faghihzadeh S. The Female Sexual Function Index (FSFI): validation of the Iranian version. FasInameh Payesh 2008; 7(2): 269-278 (in Persian).

13. Seftel AD, Padma-Nathan H, McMahan CG, et al. Male and female sexual dysfunction. Edinburgh: Mosby; 2004.

14. Kadioglu P, Yalin AS, Tiryakioglu O, et al. Sexual dysfunction in women with hyperprolactinemia: a pilot study report. J Urol 2005; 174(5): 1921-1925.

15. Atis G, Dalkilinc A, Altuntas $Y$, et al. Sexual dysfunction in women with clinical hypothyroidism and subclinical hypothyroidism. J Sex Med 2010; 7(7): 2583-2590.

16. Berman JR, Berman LA, Toler SM, et al. Safety and efficacy of sildenafil citrate for the treatment of female sexual arousal disorder: a double-blind, placebo controlled study. J Urol 2003; 170(6 Pt 1): 2333-2338.

17. Alexander MS, Rosen RC, Steinberg S, et al. Sildenafil in women with sexual arousal disorder following spinal cord injury. Spinal Cord 2011; 49(2): 273-279.

18. Sipski ML, Rosen RC, Alexander CJ, et al. Sildenafil effects on sexual and cardiovascular responses in women with spinal cord injury. Urology 2000; 55(6): 812-815.

19. Salerian AJ, Deibler WE, Vittone BJ, et al. Sildenafil for psychotropic-induced sexual dysfunction in 31 women and 61 men. J Sex Marital Ther 2000; 26(2): 133-140.

20. Cavalcanti AL, Bagnoli VR, Fonseca AM, et al. Effect of sildenafil on clitoral blood flow and sexual response in postmenopausal women with orgasmic dysfunction. Int J Gynaecol Obstet 2008; 102(2):115-119.

21. Ferrara D, Zaslau S. Success of sildenafil treatment in neurogenic female sexual dysfunction caused by L5-S1 intervertebral disk rupture: a case report. Int J Urol 2007; 14(6): 566-567.

22. Caruso S, Rugolo S, Agnello $\mathrm{C}$, et al. Sildenafil improves sexual functioning in premenopausal women with type 1 diabetes who are affected by sexual arousal disorder: a double-blind, crossover, placebo-controlled pilot study. Fertil Steril 2006; 85(5): 1496-1501.

23. Chivers M, Rosen R. Phosphodiesterase type 5 inhibitors and female sexual response: faulty protocols or paradigms? J Sex Med 2010; 7(2 Pt 2): 858-872.

24. Caruso S, Intelisano G, Lupo L, et al. Premenopausal women affected by sexual arousal disorder treated with sildenafil: a double-blind, cross-over, placebo-controlled study. BJOG 2001; 108(6): 623-628.

25. Dasgupta R, Wiseman OJ, Kanabar G, et al. Efficacy of sildenafil in the treatment of female sexual dysfunction due to multiple sclerosis. J Urol 2004; 171(3): 1189-1193.

26. Moreira SG, Brannigan JRE, Spitz A, et al. Side-effect profile of sildenafil citrate (Viagra) in clinical practice. Urology 2000; 56(3): 474-476 .

27. Bancroft J. The endocrinology of sexual arousal. J Endocrinol 2005; 186(3): 411-427.

28. D'Amati G, di Gioia CR, Bologna M, et al. Type 5 phosphodiesterase expression in the human vagina. Urology 2002; 60(1): 191-195

29. Bitzer J, Brandenburg U. Psychotherapeutic interventions for female sexual dysfunction. Maturitas 2009; 63(2): 160-163. 
30. Dennerstein L, Koochaki P, Barton I, et al. Hypoactive sexual desire disorder in menopausal women: a survey of Western European women. J Sex Med 2006; 3(2): 212-222.

31. Brozovich FV, Nicholson CJ, Degen CV, et al. Mechanisms of vascular smooth muscle contraction and the basis for pharmacologic treatment of smooth muscle disorders. Pharmacol Rev 2016; 68(2): 476-532.

32. Ichikawa T, Takao A, Manabe D, et al. The female partner's satisfaction with sildenafil citrate treatment of erectile dysfunction. Int J Urol 2004; 11(9): 75.

33. Basson R, McInnes R, Smith MD, et al. Efficacy and safety of sildenafil citrate in women with sexual dysfunction associated with female sexual arousal disorder. J Womens Health Gend Based Med 2002; 11(4): 367-377.

34. Lo Monte G, Graziano A, Piva I, et al. Women taking the "blue pill" (sildenafil citrate): such a big deal? Drug Des Devel Ther 2014; 8: 2251-2254.

35. Schoen C, Bachmann G. Sildenafil citrate for female sexual arousal disorder: a future possibility? Nat Rev Urol 2009; 6(4): $216-222$.

36. Leddy LS, Yang CC, Stuckey BG, et al. Influence of sildenafil on genital engorgement in women with female sexual arousal disorder. J Sex Med 2012; 9(10): 2693-2697.

Tables: 3

Figures: 1

References: 36

Received: 29.11.2016

Revised: 14.01.2017

Accepted: 08.02.2017

Address for correspondence:

Marzieh Akbarzadeh, MSc

Maternal - Fetal Medicine Research Center

Department of Midwifery

School of Nursing and Midwifery

Shiraz University of Medical Sciences

Shiraz

Iran

Tel.: 0711-6474250

E-mail: akbarzadm@sums.ac.ir 